\title{
Performance Evaluation of a SCM Optical Transmission System
}

\author{
Md. Shamsul Arefin ${ }^{1}$, Nusrat Tazin ${ }^{2}$, Munsury Rahman ${ }^{2}$, Tajrian Mollick ${ }^{3}$ \\ ${ }^{1}$ (Department of EEE, IBAIS University, Bangladesh) \\ ${ }^{2}$ (Core Network Department, Bangla Trac Communication Limited, Bangladesh) \\ ${ }^{3}$ (Department of EEE, Bangladesh University, Bangladesh)
}

\begin{abstract}
This paper focuses on the impact of Optical Beat Interference (OBI) on the performance of subcarrier multiplexed optical transmission system for the application on radio link via optical fiber. Performance results are evaluated for QPSK modulation of Microwave subcarriers with digital NRZ coded random data patterns. A suitable bandwidth of $890-950 \mathrm{MHz}$ is selected for subcarriers and channel bandwidth of $200 \mathrm{KHz}$ and carrier separation of $250 \mathrm{KHz}$ are considered. By measuring the Optical Bit Interference (OBI) performance limitations of the subcarrier multiplexing optical transmission system is investigated. The OBI for 10 channels for input power $1 \mathrm{~dB}$ is $-40 \mathrm{~dB}$ whereas for 110 channels the OBI is $-20 \mathrm{~dB}$. The SIR is also investigated to evaluate the performance. The achievable SIR for NRZ-QPSK subcarrier multiplexed transmission system is about $5 \mathrm{~dB}$ corresponding to 110 channels whereas the corresponding SIR is only $28 \mathrm{~dB}$ for 10 channels for input power 1 $\mathrm{dB}$. The SIR also degrades as the input power is increased. Investigation of BER shows that for 10 channels the BER is $10^{-2}$ whereas for 110 channels BER is around $10^{-2}$. This performance also degrades as the input power is increased to $20 \mathrm{~dB}$.
\end{abstract}

Keywords: BER, OBI, SCM, SNR, SMF, SIR, WDM.

\section{INTRODUCTION}

In this section, introduction to optical fibre communication, the role of multiplexing schemes especially subcarrier multiplexing (SCM) and wave division multiplexing (WDM) in communication system and the purposes and objectives of this work are discussed.

\subsection{Introduction to Fiber Optic Communication \& Multiplexing Schemes}

The world of communications has been revolutionized by the appearance of light wave technology. The key to the great success of optical communications lies with the enormous potential bandwidth (in the range of tens of Terahertz) and low transmission loss of optical fibers. In order to maximize the information transfer over any communication link, it is usual to multiplex several signals onto the transmission medium. Multiplexing schemes are related to multiple-access schemes, as they provide a way for several users to access a system.

\subsubsection{Fiber Optic Communication}

Fiber-optic communication is a method of transmitting information from one place to another by sending pulses of light through an optical fiber. Optical fiber is used by many telecommunication companies to transmit telephone signals, Internet communication, and cable television (CATV) signals. Due to much lower attenuation and interference, optical fiber has large advantages over existing copper wire in long-distance and high-demand applications. However, infrastructure development within cities was relatively difficult and timeconsuming, and fiber-optic systems were complex and expensive to install and operate. Due to these difficulties, fiber-optic communication systems have primarily been installed in long-distance applications, where they can be used to their full transmission capacity, offsetting the increased cost.

The process of communicating using fiber-optics involves the following basic steps: Creating the optical signal involving the use of a transmitter, relaying the signal along the fiber, ensuring that the signal does not become too distorted or weak, receiving the optical signal, and converting it into an electrical signal. Modern fiber-optic communication systems generally include an optical transmitter to convert an electrical signal into an optical signal to send into the optical fiber, a cable containing bundles of multiple optical fibers that is routed through underground conduits and buildings, multiple kinds of amplifiers, and an optical receiver to recover the signal as an electrical signal. The information transmitted is typically digital information generated by computers, telephone systems, and cable television companies.

\subsubsection{Multiplexing Schemes}

With the development of an information-oriented society and the explosive growth of the Internet, the requirements of network capacity have increased dramatically, which is promoting the construction of a 
broadband trunk network. In order to use the optical bandwidth provided by optical fibers more efficiently, new transmission technologies have been developed in recent years, such as time division multiplexing (TDM), frequency division multiplexing (FDM), wavelength division multiplexing (WDM), sub-carrier multiplexing (SCM) etc and their combinations. Apart from noise accumulation, high-speed TDM optical systems suffer from chromatic dispersion, nonlinear crosstalk, and polarization-mode dispersion (PMD). Optical systems with data rates of $10 \mathrm{~Gb} / \mathrm{s}$ and higher require precise dispersion compensation and careful link engineering. The increase of bandwidth demand is linear with the supply of network services in many cellular operators. The network is setup to provide the user the services such as video streaming, data communication, push email, teleconference, mobile banking, etc. that require large bandwidth in the traffic. Therefore, the need of broadband consumption of a user has increased. In order to supply the need of bandwidth, many researchers currently actively investigate and focus on three main components; spectrum allocation of the frequency band, efficiency and increase of capacity of the cell. Thus, the combination of SCM/WDM is expected to supply the demand of increased bandwidth for the cellular communication. For simultaneously accessible subcarrier channels the capacity should ideally increase linearly with the number of subcarrier channels.

SCM is a scheme where multiple signals are multiplexed in the radio frequency (RF) domain and transmitted by a single wavelength. The most significant advantage of SCM in optical communications is its ability to place different optical carriers together closely. On the other hand, WDM is a multiplexer at the transmitter to join the signals together, and a de-multiplexer at the receiver to split them apart. In WDM each laser is modulated at a given speed, and the total aggregate capacity being transmitted along the high-bandwidth fiber is the sum total of the bit rates of the individual lasers. A significant advantage of SCM is that microwave devices are more mature than optical devices; the stability of a microwave oscillator and the frequency selectivity of a microwave filter are much better than their optical counterparts. In addition, the low phase noise of RF oscillators makes coherent detection in the RF domain easier than optical coherent detection, and advanced modulation formats can be applied easily. A popular application of SCM technology in fiber optic systems is analog CATV distribution. Because of the simple and low-cost implementation, SCM has also been proposed to transmit multichannel digital optical signals using direct detection for local area optical networks.

Wavelength division multiplexing (WDM) is a promising approach that can use the enormous bandwidth of the optical fiber. WDM technology spreads transmission capacity into various wavelength channels and uses relatively low data rates at each wavelength. A single fiber can be employed for multiple data streams simultaneously. All-optical networks employing the concept of WDM and wavelength routing are considered as the transport networks for the future. In such networks, two adjacent nodes are connected by one or multiple fibers. Each node has a dynamically configurable optical switch which supports fiber switching and wavelength switching, i.e., the data on a specified input fiber and wavelength can be switched to a specified output fiber on the same wavelength. In order to transfer data between source-destination node pairs, a light path needs to be established by allocating the same wavelength throughout the route of the transmitted data. However, due to the selectivity of optical filters and limitations in the wavelength stability of semiconductor lasers, the minimum channel spacing is $50 \mathrm{GHz}$ in current commercial WDM systems. Narrower channel spacing has been pursued by industry and the research community to increase fiber transmission capacity. More sophisticated modulation formats may help to increase the bandwidth efficiency compared to the basic ON-OFF keying modulation.

\subsection{Recent Researches}

With the development of optical fiber communication technology, there had been many researches and thesis on the development of multiplexing systems. The researches on Sub-carrier multiplexing were focused on the fiber modes, types of detection schemes, interference, noises and overall performances evaluating signal to noise ratio (SNR). Various issues for radio over fiber communication links were investigated in order to provide a cost-effective, high performance solution for high speed data rates by the available bandwidth of the electrical and optical components in the thesis "The SCM/WDM system model for radio over fiber communication link" [1]. SCM must be used in conjunction with WDM to utilize any significant fraction of the fiber bandwidth. The results presented higher bandwidth for long distance communication system (SMF, $150 \mathrm{~km}$ ) by using SCM/WDM for Radio over Fiber. Therefore, the efficiency of bandwidth utilization of SCM is expected to be much better than conventional optical WDM.

A dissertation focused on the use of subcarrier multiplexing (SCM) in multimode fibers, utilizing carrier frequencies above what is generally utilized for multimode fiber transmission, to achieve high bit rates in "Modeling, analysis and design of subcarrier multiplexing on multimode fiber" [2]. In the high frequency region, the magnitude response of multimode fiber does not decrease monotonically as a function of the frequency but is shown to become relatively flat (but with several deep nulls) with the amplitude below that at DC. The statistical properties of this frequency response at high frequencies were analyzed in that dissertation. The probability density function of the magnitude response at high frequencies was found to be a Rayleigh 
density function. The average amplitude in this high frequency region does not depend on the frequency but depends on the number of modes supported by the fiber. To transmit a high bit rate signal over the multimode fiber, subcarrier multiplexing was adopted. The performance of the SCM multimode fiber system was presented. The performance of the SCM system was significantly degraded if there were some subcarriers located at the deep nulls of the fiber. Equalization and spread spectrum techniques were investigated but were shown to be not effective in combating the effects of these nulls. To cancel the effects of these deep nulls, training process and diversity coding are considered. The practical limits and capacity of the SCM multimode fiber system are investigated. It was shown that a signal with a bit rate of $1.45 \mathrm{Gbps}$ can be transmitted over a distance up to 5 $\mathrm{km}$. An error-free four-user $\sim 3-\mathrm{GHz}$ spaced SCM experiment were demonstrated, where, for the first time, optical direct-detection via optical hyperfine wavelength-division multiplexing was used instead of heterodyne beating detection that is conventional for SCM in "Four-User 3-GHz-Spaced Subcarrier Multiplexing (SCM) Using Optical Direct-Detection via Hyperfine WDM" [3] . A hyperfine optical blocking filter allowed optical single-sideband modulation with strong carrier suppression $>20 \mathrm{~dB}$ below the unsuppressed single sideband and strong sideband suppression $>20 \mathrm{~dB}$. Subcarrier multiplexing by chaotic multi-tone modulation was investigated in the thesis "Subcarrier Multiplexing by Chaotic Multi tone Modulation" [4]. It is found that optical chaotic light coupled be achieved by injecting multiple subcarriers into a self-pulsating laser diode. Synchronization between two identical chaotic systems (drive and response) could be implemented provided that the conditional Lyapunov exponents were all negative. By adding amplitude modulation (AM) signals to each subcarrier, the two systems became asymptotically synchronized. The AM signals in each subcarrier could be recovered by the introduction of a filtering process where the Lyapunov exponent of the synchronized error function matches the cut off frequency of a first order low pass filter. A general model of optical beat interference in presence of Four-Wave Mixing (FWM) was presented in the paper "Effect of Optical Beat Interference in SCM/WDM Optical Networks in Presence of FWM" [5]. This research finds that SCM with WDM can be used to increase the capacity of any optical network. For simultaneously accessible subcarrier channels the capacity should ideally increase linearly with the number of subcarrier channels. However, when two lasers carrying subcarrier channel multiplexed data operate with much closed spaced wavelength, beating between the lasers and between the lasers and Four-Wave mixing terms can occur. This will increase the noise at the photodetector. Computer simulations for externally modulated single-mode laser transmitted through a Dispersion Shifted Fiber (DSF) were presented there. For degenerate case, the results showed degradation in Carrier to Interference Ratio (CIR) when the laser frequency separation was equal to and half of the subcarrier channel frequency.

The paper "Performance Evaluation of SCM-WDM System Using Different Linecoding" [6] investigated the theoretical performance analysis for a SCM optical transmission system in presence of optical beat interference $(\mathrm{OBI})$ which occurs during the photo detection process.

\subsection{Purposes and Objectives of the work}

In our works, we evaluated the performance of SCM communication system for a number of channels and the Optical Bit Interference (OBI), Signal to Interference Ratio (SIR) and Bit Error Rate (BER) are measured to find the suitable design of a network for a given bandwidth. The main purpose and objectives of this work are listed as follows:

- Theoretical performance analysis for SCM system in presence of Optical Bit Interference (OBI).

- Measure and plot graphical representation of OBI vs Number of channels in order to evaluate the performance of the system with increasing number of channels.

- Measure and plot the graphical representations of Number of channels vs SIR to show the relative performance and conditions for OBI.

- Measure and plot the graphical representation of BER vs Number of channels.

- Find out the suitable system design for a given bandwidth of $890 \mathrm{MHz}$ to $960 \mathrm{MHz}$.

\section{OPTICAL FIBER COMMUNICATION}

Now we are in the twenty first century, the era of 'Information Technology'. There is no doubt that information technology has had an exponential growth through the modern telecommunication systems. Particularly, optical fiber communication plays a vital role in the development of high quality and high-speed telecommunication systems. Today, optical fibers are not only used in telecommunication links but also used in the Internet and local area networks (LAN) to achieve high signalling rates.

\subsection{Structure of Optical Fiber}

Optical fiber is a dielectric waveguide or medium in which information (voice, data or video) is transmitted through a glass or plastic fiber, in the form of light. The basic structure of an optical fiber is shown in fig. 1. It consists of a transparent core with a refractive index $\mathrm{n}_{1}$ surrounded by a transparent cladding of a slightly less refractive index $\mathrm{n}_{2}$. The refractive index of cladding is less than $1 \%$, lower than that of core. Typical 
values for example are a core refractive index of 1.47 and a cladding index of 1.46. The cladding supports the waveguide structure, protects the core from absorbing surface contaminants and when adequately thick, substantially reduces the radiation loss to the surrounding air. Glass core fibers tend to have low loss in comparison with plastic core fibers. Additionally, most of the fibers are encapsulated in an elastic, abrasionresistant plastic material which mechanically isolates the fibers from small geometrical irregularities and distortions. A set of guided electromagnetic waves, also called the modes of the waveguide, can describe the propagation of light along the waveguide. Only a certain number of modes are capable of propagating through the waveguide. [7]

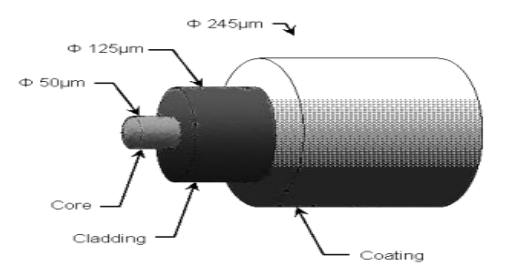

Fig. 1: Cross section of Optical Fiber.

\subsection{General Overview of Optical Fiber Communication System}

Like all other communication system, the primary objective of optical fiber communication system also is to transfer the signal containing information (voice, data, video) from the source to the destination. The general block diagram of optical fiber communication system is shown in the fig. 2. The source provides information in the form of electrical signal to the transmitter. The electrical stage of the transmitter drives an optical source to produce modulated light wave carrier. Semiconductor LASERs or LEDs are usually used as optical source here. The information carrying light wave then passes through the transmission medium i.e. optical fiber cables in this system. Now it reaches to the receiver stage where the optical detector demodulates the optical carrier and gives an electrical output signal to the electrical stage. The common types of optical detectors used are photodiodes (p-i-n, avalanche), phototransistors, photoconductors etc. Finally the electrical stage gets the real information back and gives it to the concerned destination.

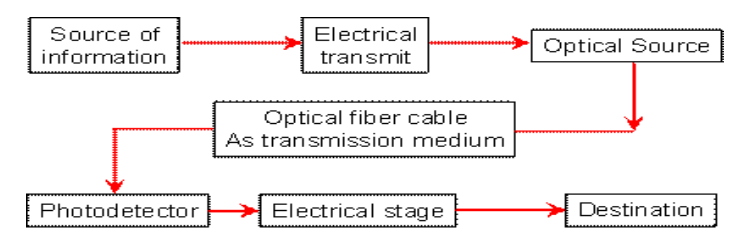

Fig. 2: Optical Fiber Communication System

It is notable that the optical carrier may be modulated by either analog or digital information signal. In digital optical fiber communication system the information is suitably encoded prior to the drive circuit stage of optical source. Similarly at the receiver end a decoder is used after amplifier and equalizer stage.

\subsection{Primary Elements of Optical Fiber Communication System}

Fig. 3 shows the major elements used in an optical fiber communication system. As we can see the transmitter stage consists of a light source and associated drive circuitry. Again, the receiver section includes photodetector, signal amplifier and signal restorer. Additional components like optical amplifier, connectors, splices and couplers are also there. The regenerator section is a key part of the system as it amplifies and reshapes the distorted signals for long distance links.

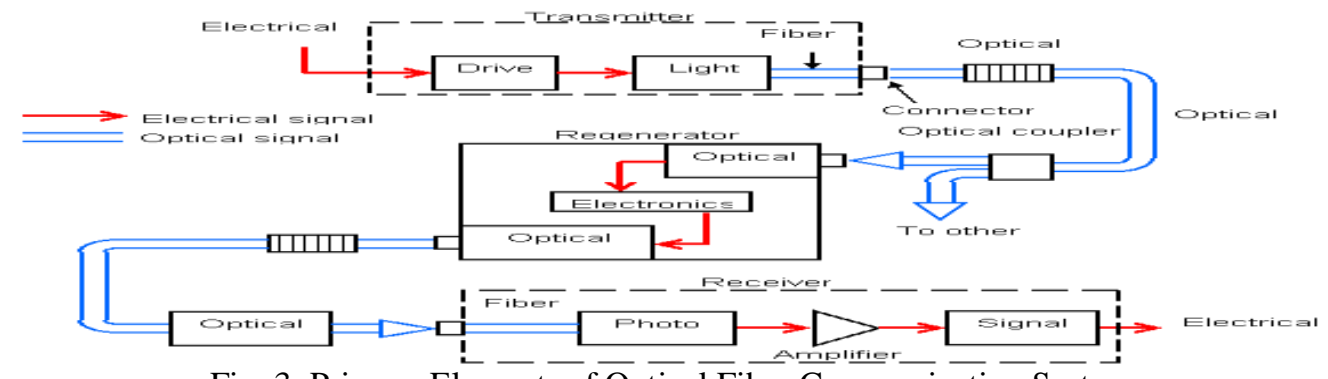

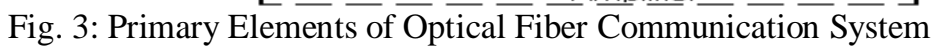




\subsection{Generations of Optical Fiber Communications}

Table 1 shows the different generations of optical fiber communication. In generation I, mostly GaAs based LEDs and laser diodes having emission wavelength $0.8 \mu \mathrm{m}$ were used. From 1974 to 1978, graded index multimode fibers were used. From 1978 onwards, only single mode fibers are used for long distance communication. During the second generation the operating wavelength is shifted to $1.3 \mu \mathrm{m}$ to overcome loss and dispersion. Further InGaAsP hetero-junction laser diodes are used as optical sources. In the third generation the operating wavelength is further shifted to $1.55 \mu \mathrm{m}$ and the dispersion-shifted fibers are used. Further single mode direct detection is adopted. In the fourth generation erbium doped optical (fiber) amplifiers are fabricated and the whole transmission and reception are performed only in the optical domain. WDM is introduced to increase the bit rate. In the proposed next generation ( $\mathrm{V}$ generation), soliton based lossless and dispersion less optical fiber communication will become a reality. At that time, the data rate may increase beyond $1000 \mathrm{~Tb} / \mathrm{s}$.

Table 1: Generations of optical fiber communication systems

\begin{tabular}{|c|c|c|c|c|c|}
\hline GENERATION & $\begin{array}{c}\text { WAVELENGTH } \\
\text { OF OPTICAI } \\
\text { SOURCE }(\mathrm{um})\end{array}$ & $\begin{array}{c}\text { BIT RATE } \\
(\mathrm{Mb} / \mathrm{s})\end{array}$ & $\begin{array}{c}\text { REPEATER } \\
\text { SPACING } \\
(\mathrm{km})\end{array}$ & $\begin{array}{c}\text { LOSS } \\
(\mathrm{dB} / \mathrm{km})\end{array}$ & $\begin{array}{c}\text { EXISTED } \\
\text { UPTO }\end{array}$ \\
\hline I & 0.8 & 4.5 & 10 & 1 & 1980 \\
\hline II & 1.3 & $1.7 \times 10^{2}$ & 50 & $<1$ & 1987 \\
\hline III & 1.55 & $1.0 \times 10^{4}$ & 70 & $<0.2$ & 1990 \\
\hline IV & 1.55 & $1.0 \times 10^{5}$ & 100 & $<0.002$ & 2000 \\
\hline V & 1.55 & $>1.0 \times 10^{9}$ & $>100$ & $<0.002$ & \\
\hline
\end{tabular}

\section{SUB-CARRIER MULTIPLEXING (SCM) OPTICAL TRANSMISSION}

More recently, however, the utilization of substantially higher frequency microwave subcarriers multiplexed in the frequency domain before being applied to intensity modulate a high speed injection laser source has generated significant interest. Such microwave subcarrier multiplexing (SCM) enables multiple broadband signals to be transmitted over single-mode fiber and appears particularly attractive for video distribution systems. In addition, with SCM, conventional microwave techniques can be employed to subdivide the available intensity modulation bandwidth in a convenient way. The result is a useful multiplexing technique which does not require sophisticated optics or source wavelength specification. Either digital or analog modulation of the subcarriers can be utilized by up converting to a narrowband channel at high frequency employing either amplitude, frequency or phase shift keying (i.e. ASK, FSK or PSK), and either amplitude, frequency or phase modulation (i.e. AM,FM or PM) respectively. For digital signals, FSK has the advantage of being simple to implement, both at the modulation and demodulation, whereas for analog video signals the modulations of the high frequency carrier (up conversion) is often carried out using either AM-VSB (vestigial sideband) or FM techniques. In both cases, the multicarrier signal is formed by frequency division multiplexing (FDM) of the modulated microwave subcarriers in the electrical domain prior to conversion to an intensity modulated optical signal.

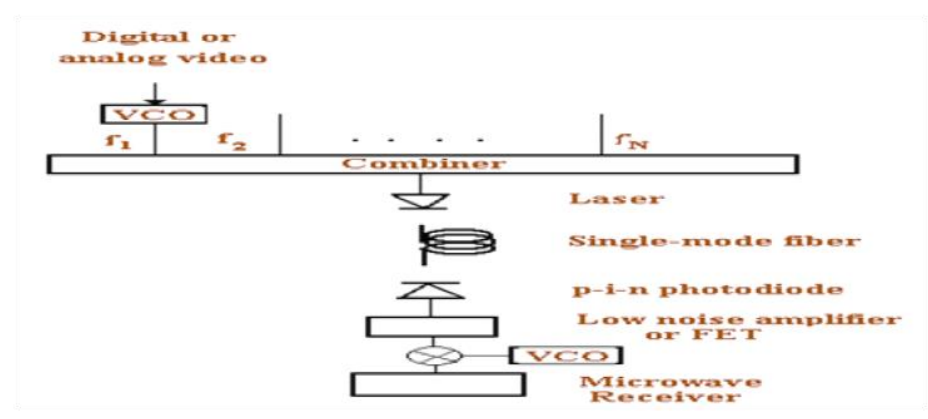

Fig. 4: Basic sub-carrier multiplexed (SCM) fiber system. 


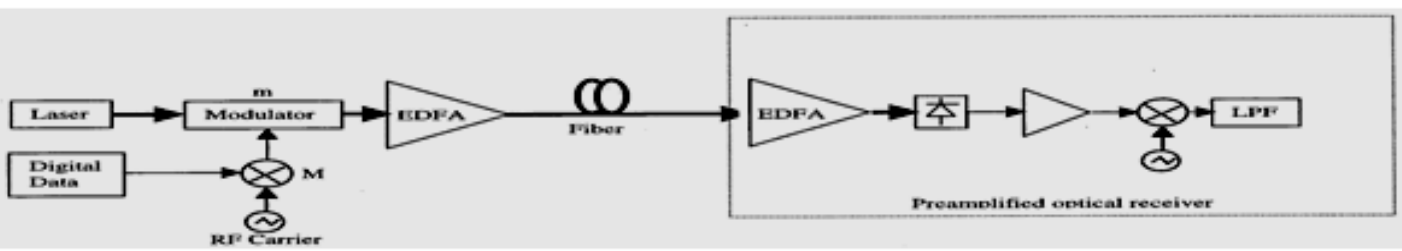

Fig. 5: SCM-WDM system architecture.

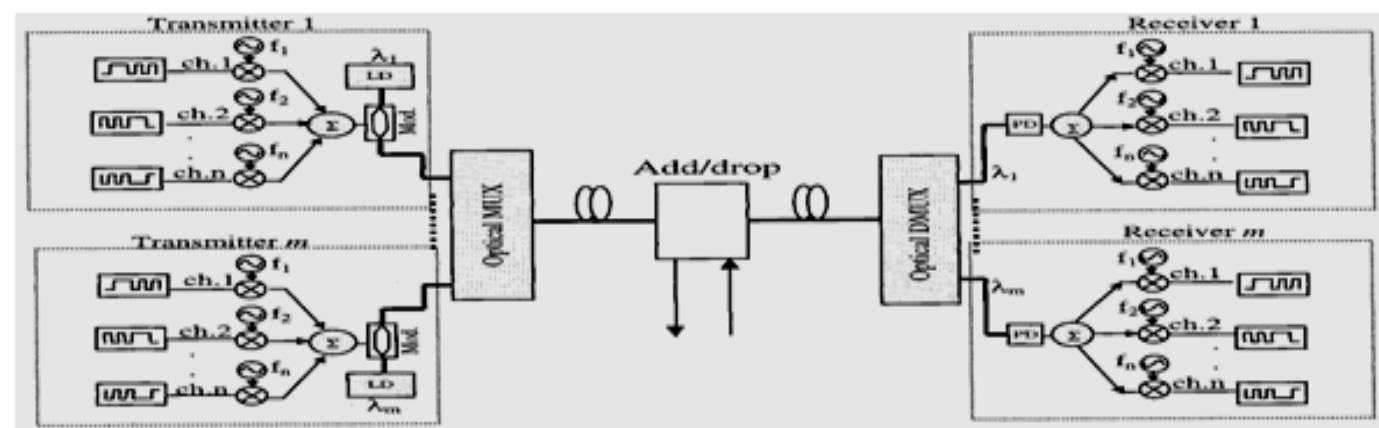

Fig. 6: Simplified block diagram of SCM system with amplified optical receiver.

\subsection{Limitations of SCM (Crosstalk)}

The term crosstalk refers to any phenomenon by which a signal transmitted on one circuit or channel of a transmission system creates an undesired effect in another circuit or channel. Crosstalk is usually caused by undesired capacitive, inductive, or conductive coupling from one circuit, part of a circuit, or, channel to another.[19] Crosstalk will be one of the major limitations for the introduction of OXC in all optical networks. Crosstalk $=\frac{P_{i o}^{\text {out }(\text { ref })}-P_{i o 1}^{\text {out }}}{P_{\text {io }}^{\text {out }(\text { ref })}}$

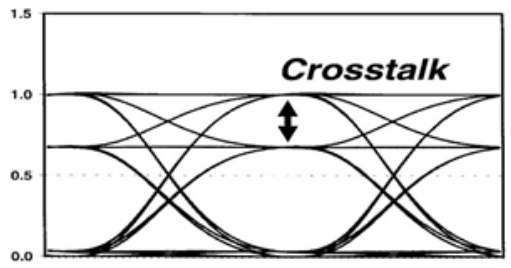

Fig. 7: Generation of Crosstalk represented by Eye-Diagram.

The narrow channel spacing in WDM links give rise to crosstalk. Crosstalk can be introduced by almost any components in WDM system, including optical filters, wavelength MUX \& DEMUX, optical switches, optical amplifiers and the fiber itself. In telecommunication, crosstalk is often distinguishable as pieces of speech or signalling tones leaking from other people's connections. If the connection is analog, twisted pair cabling can often be used to reduce the effects of cross-talk. Alternatively, the signals can be converted to digital form, which is much less susceptible to cross-talk. In wireless telecommunication, cross-talk is often denoted co-channel interference, and is related to adjacent channel interference. Crosstalk normally refers to a signal affecting another nearby signal. Usually the coupling is capacitive, and to the nearest neighbour, but other forms of coupling and effects on signal further away are sometimes important, especially in analog designs.

\subsection{Types of cross talk}

\subsubsection{Crosstalk due to Filtering}

Crosstalk element superimposed on the signal as a random power adding process. However, there is no significant beating element. A narrow band optical filter would largely reduce the electrical impairment.

\subsubsection{Linear Crosstalk}

- Space switches crosstalk.

- Homowavelength crosstalk.

- Heterowavelength crosstalk.

Both arise due to non-ideal wavelength filtering, occurrence in multiplexer, de-multiplexer, filter and space switches.

\subsubsection{Non-linear Crosstalk}


- Four wave mixing: Wave mixing to give rise of new frequency.

- Cross phase modulation: Intensity dependent refractive index.

- Scattering: Transfer of power between propagation modes.

\subsubsection{Co-Channel Interference (CCI)}

$\mathrm{CCI}$ is crosstalk from two different radio transmitters using the same frequency. There can be several causes of co-channel radio interference. There examples are given below:

- Adverse weather conditions.

- Poor frequency planning.

- Overly-crowded radio spectrum.

\subsubsection{Adjacent Channel Interference (ACI)}

Adjacent channel interference (ACI) is distinguished from crosstalk. It is the interference caused by extraneous power from a signal in an adjacent channel. Adjacent channel interference may be caused by inadequate filtering. Such as incomplete filtering of unwanted modulation products in Frequency Modulation (FM) systems, improper tuning, or poor frequency control, in either the reference channel or the interference channel, or both.

\section{PERFORMANCE EVALUATION OF SCM}

Optical transmission with subcarrier multiplexing (SCM) is a scheme where multiple signals are multiplexed in the radio frequency (RF) domain and are used to modulate directly a laser diode. A significant advantage of SCM is that microwave devices are more mature than optical devices; the stability of a microwave oscillator and the frequency selectivity of a microwave filter are much better than their optical counterparts. In addition, the low phase noise of RF oscillators makes coherent detection in the RF domain easier than optical coherent detection, and advanced fiber optic systems is analog cable television (CATV) distribution. Because of the simple and low cost implementation, SCM has also been proposed to transmit multi-channel digital signals using direct detection for local area optical networks.

How OBI limits the number of subcarriers or in other words the number of subcarriers in SCM system is analysed in this paper. The square law envelope detection by the nonlinear device photodetector (PD) causes OBI. The function of the PD in one of the user receivers is considered. A total of $\mathrm{M}$ optical fields are present at PD input. At the output of the PD, we would expect $\mathrm{M}$ terms representing the field intensities, plus M (M-1) cross term. If the spectral content of any of these cross terms falls within the passband of the bandpass filter (BPF) in the intended user channel, it will cause interference. As will be shown, OBI can represent a serious limitation on the transmission capacity. This paper includes the performance limitation of SCM due to this OBI.

\subsection{Theoretical Analysis of SCM in Presence of OBI}

There are $\mathrm{M}$ numbers of subcarriers in a given optical channel, having the same average power. Each of these fields can be represented by:

$\mathbf{e}_{\mathbf{i}}(\mathbf{t})=\sqrt{\mathbf{s}_{\mathbf{i}}(\mathbf{t})}$

Where $S_{i}$ is the intensity modulation by an RF signal of center frequency $f_{i}$ and can be represented by:

$S_{i}=1+m(t) \cos \left(2 \pi f_{i} t\right)$

Where $m(t)$ is NRZ data signal with bit period $T_{b}$.

The total field in an optical channel is the sum of $\mathrm{M}$ fields and can be represented as:

$\mathbf{e}_{\mathrm{in}}(\mathbf{t})=\sum_{\mathrm{i}=\mathbf{1}}^{\mathrm{M}} \mathbf{e}_{\mathrm{i}}(\mathbf{t})$

The electric field at the output of the fiber is given by:

$\mathbf{e}_{\mathbf{0}}(\mathbf{t})=\left[\mathbf{e}_{\mathrm{in}}(\mathbf{t}) * \mathbf{h}_{\mathrm{f}}(\mathbf{t})\right] \mathrm{e}^{-\alpha L}$

Where $\alpha$ is the fiber attenuation coefficient, $L$ is the fiber length and $h_{f}(t)$ represents the fiber impulse response.

The photodetector $(\mathrm{PD})$ converts this field into an electrical signal proportional to the field intensity. Photodetector output current is then given by:

$\mathbf{i}(\mathbf{t})=\mathbf{R}\left|\mathbf{e}_{\mathbf{o}}(\mathbf{t})\right|^{2}+\mathbf{n}(\mathbf{t})$

Where $\mathrm{R}$ is the responsivity of the $\mathrm{PD}$ and $\mathrm{n}(\mathrm{t})$ represents the noise due to photodetector and preamplifier.

By expanding equation (5), signal component and cross component of photodetector output can be expressed as:

$\mathbf{i}_{\mathbf{s}}(\mathbf{t})=\left\{\sum_{\mathrm{i}=\mathbf{1}}^{\mathrm{M}} \mathbf{e}_{\mathbf{0}}{ }^{2}(\mathbf{t})\right\}$

$$
\mathbf{i}_{\mathbf{c}}(\mathbf{t})=\left\{\mathbf{2} \sum_{\mathbf{i}=\mathbf{1}}^{\mathrm{M}} \sum_{\mathrm{l}=\mathbf{i}+\mathbf{1}}^{\mathrm{M}} \mathbf{e}_{\mathbf{i}}(\mathbf{t}) \mathbf{e}_{\mathbf{l}}(\mathbf{t})\right\}
$$

Here $i_{c}(t)$ contributes nonzero beat interference terms. The output of the PD is passed through a preamplifier followed by a band pass filter. If any of the spectral components of $i_{c}(t)$ falls within the bandwidth of any of the M users BPF, it will cause OBI.

The frequency spectrum of the desired i-th subcarrier channel signal component can be obtained by taking fourier transform of $i_{s}(t)$ as: 


$$
\begin{aligned}
& \mathbf{i}_{s}(\mathbf{i})(\mathbf{f})=\int_{-\infty}^{+\infty} \mathbf{i}(\mathbf{i})(t) e^{(-\mathrm{jft})} \\
& =2 \pi \delta(f)+(\mathbf{T} / 2) *\left[\operatorname{sinc} \mathbf{T}\left(\mathbf{f}-\mathbf{f}_{\mathrm{i}}\right)+\operatorname{sinc} \mathbf{T}\left(\mathbf{f}+\mathbf{f}_{\mathrm{i}}\right)\right] \mathrm{e}^{(-\mathrm{j} \mathbf{f} \mathrm{T} / 2)}
\end{aligned}
$$

Where $\mathrm{f}_{\mathrm{i}}$ represents the required subcarrier frequency.

The power spectrum of the i-th subscriber's signal component can be expressed as:

$$
\begin{aligned}
P_{s}(i)(f) & =\left[i_{s}(i)(f)\right]^{2} \\
& =4 \pi 2 \delta(f)+(\mathbf{T} 2 / 4) *\left[\operatorname{sinc}^{2} \mathbf{T}\left(\mathbf{f}-\mathbf{f}_{\mathrm{i}}\right)+\operatorname{sinc}^{2} \mathbf{T}\left(\mathbf{f}+\mathbf{f}_{\mathrm{i}}\right)\right] \mathrm{e}^{(-\mathrm{jfr} / 2)}
\end{aligned}
$$

Using band pass filter, output signal power of the required sub-carrier can be calculated as:

$$
\begin{aligned}
\mathbf{P i}(\mathbf{s i g}) & =\int_{\mathbf{f}_{i}-\mathbf{B} / 2}^{f_{\mathbf{f}+\mathbf{B}} / \mathbf{P}} \mathbf{P}_{\mathbf{s}(\mathbf{i})}(\mathbf{f}) \mathbf{d f} \\
& =\int_{\mathbf{f}_{\mathbf{i}-\mathbf{B} / 2}}^{f_{\mathrm{i}}+\mathbf{2}}(\boldsymbol{\tau} \mathbf{2} / \mathbf{4}) *[\operatorname{sinc} 2 \tau(\mathbf{f}-\mathbf{f i})] \mathbf{d f}
\end{aligned}
$$

Where $B$ is the specified bandwidth of the subcarrier or bandwidth of the BPF. Here sinc2 $T(f+f i), \delta(f)$ terms fall outside the range of integration.

Similarly, the frequency spectrum of the composite cross component can be expressed as:

$\mathbf{i}_{\mathbf{c}}(\mathbf{i})(\mathbf{f})=\int_{-\infty}^{+\infty} \mathbf{i}(\mathbf{i})(t) e^{(-j f t)}$

The power spectrum of the composite cross component can be expressed as:

$\mathbf{P}_{\mathbf{c}}(\mathbf{i})(\mathbf{f})=\left[\mathbf{i}_{\mathbf{c}}(\mathbf{i})(\mathbf{f})\right]^{2}$

The cross component present in the required bandwidth $B$ is:

$\mathbf{P}_{\mathrm{i}}(\mathbf{c r o s s})=\int_{\mathrm{f}_{\mathrm{i}-\mathrm{B} / 2}}^{\mathrm{f}_{\mathrm{i}}+\mathrm{B} / \mathbf{2}} \mathbf{P}_{\mathbf{c}(\mathrm{i})}(\mathrm{f}) \mathrm{df}$

This $\mathrm{P}_{\mathrm{i}}$ (cross) is the source of the OBI.

$\mathbf{S N R}=\mathbf{P}_{\mathrm{i}}($ sig $) / \mathbf{P}_{\mathrm{i}}($ cross $)$

$\operatorname{BER}=0.5 \operatorname{erfc}(\sqrt{2 \times S N R})$

\subsection{Plots and Observations}

A theoretical performance analysis is presented for a subcarrier multiplexed (SCM) optical transmission system in presence of Optical Bit Interference (OBI) which occurs during the photo detection process for nonreturn to zero (NRZ) line coding. A multiuser SCM system for a suitable bandwidth is investigated using MATLAB and in this investigation allocated bandwidth for each user was $200 \mathrm{KHz}$ with carrier separation 200 KHz. The results are shown below from fig. 8 to fig. 11. The modulated RF carriers in SCM system then intensity modulate each optical carrier in WDM systems. Here we have used three types of line coding schemes NRZ, Manchester and Miller coding. Spectrum of signal and cross components respectively obtained using MATLAB. The signal and cross components were estimated using a 131072 point of FFT. Here we have plotted the comparative representation of SNR versus number of channels/subcarriers with NRZ coding.

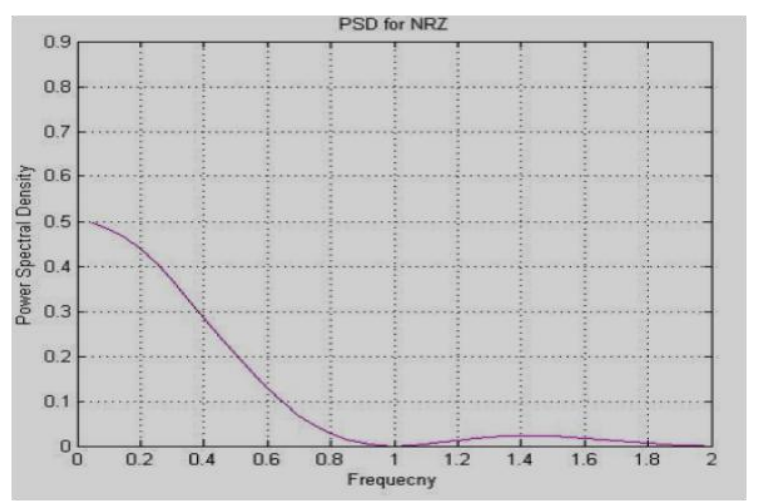

Fig. 8: Plots of PSD of signal vs frequency for NRZ data. 


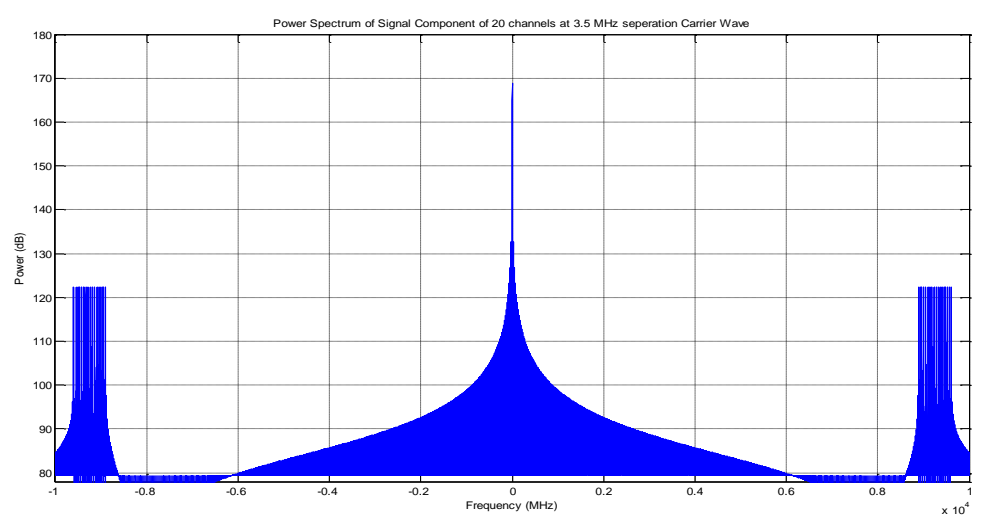

Fig. 9: Plots of power spectrum of signal component of 20 channels at $3.5 \mathrm{MHz}$ carrier separation of an SCM system for NRZ data.

Observation: The above fig. 9 shows the plot of power spectrum of signal component for 20 channels at 3.5 $\mathrm{MHz}$ carrier separation. Only noise of the SCM system is present with the signal component, thereby the amplitude is less.

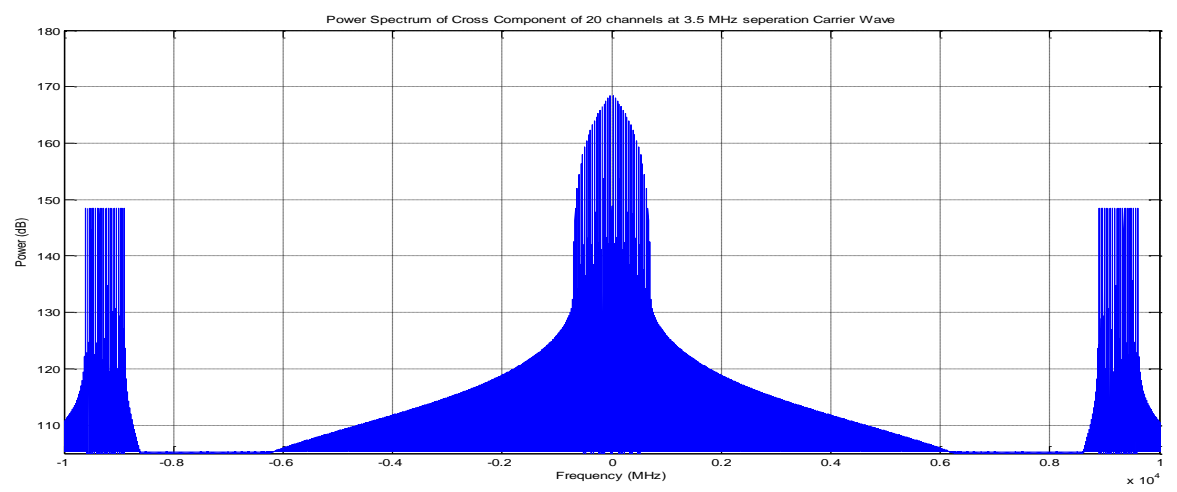

Fig. 10: Plots of power spectrum of cross component of 20 channels at $3.5 \mathrm{MHz}$ carrier separation of an SCM system for NRZ data.

Observation: The above fig. 10 shows the plot of power spectrum of cross component for 20 channels at 3.5 $\mathrm{MHz}$ carrier separation. Optical bit interference (OBI) is present with noise of the SCM system; thereby the amplitude of the interference plus noise is more.

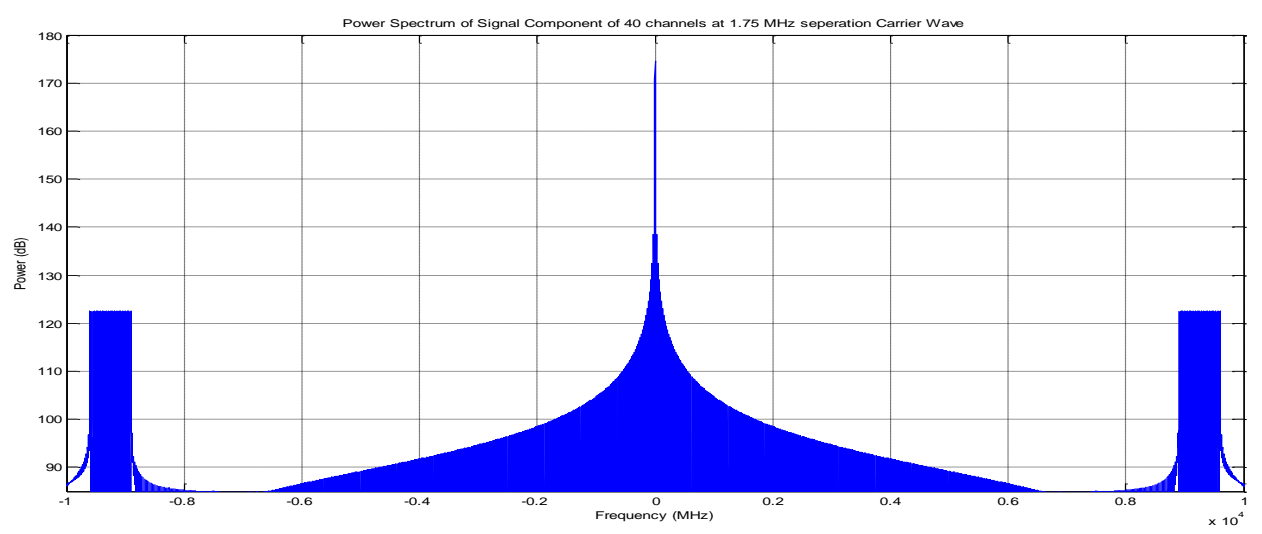

Fig. 11: Plots of power spectrum of signal component of 40 channels at $1.75 \mathrm{MHz}$ carrier separation of an SCM system for NRZ data.

Observation: The above fig. 11 shows the plot of power spectrum of signal component for 40 channels at 1.75 $\mathrm{MHz}$ carrier separation. Only noise of the SCM system is present with the signal component, thereby the amplitude is less. 


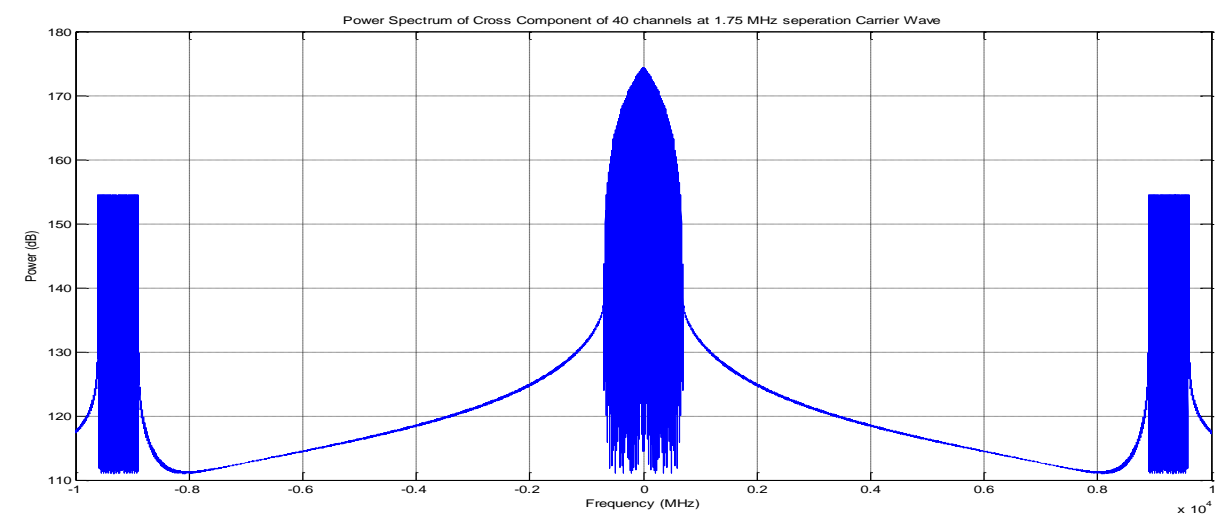

Fig. 12: Plots of power spectrum of cross component of 20 channels at $3.5 \mathrm{MHz}$ carrier separation of an SCM system for NRZ data.

Observation: The above fig. 12 shows the plot of power spectrum of cross component for 40 channels at 1.75 $\mathrm{MHz}$ carrier separation. Optical bit interference (OBI) is present with noise of the SCM system; thereby the amplitude of the interference plus noise is more. Moreover there is significant presence of interference is seen in the power spectrum plot.

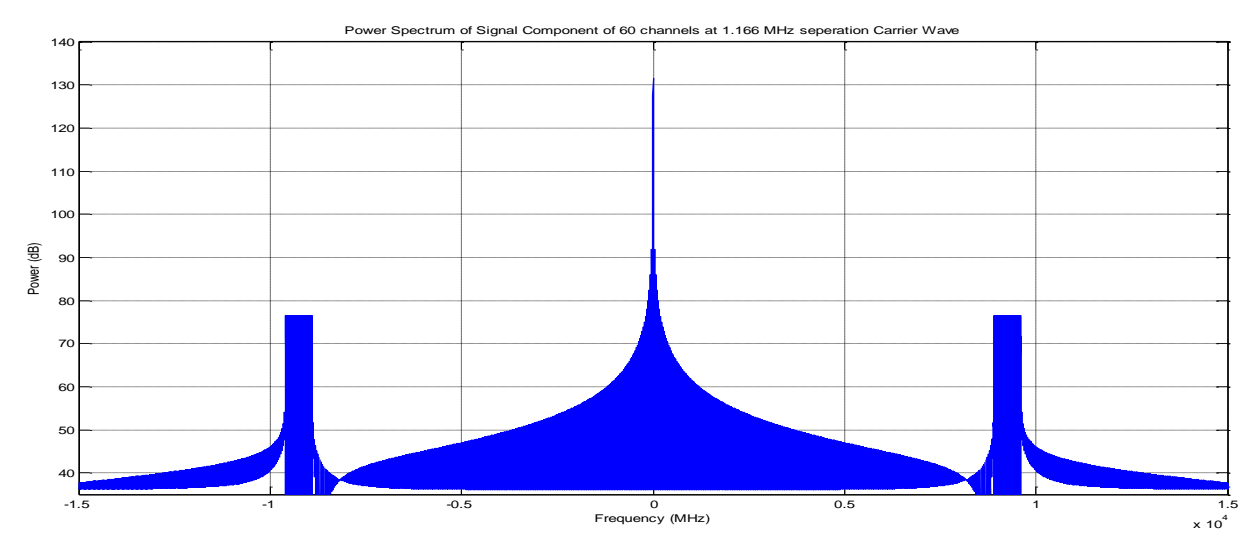

Fig. 13: Plots of power spectrum of signal component of 60 channels at $1.166 \mathrm{MHz}$ carrier separation of an SCM system for NRZ data.

Observation: The above fig. 13 shows the plot of power spectrum of signal component for 60 channels at 1.166 $\mathrm{MHz}$ carrier separation. Only noise of the SCM system is present with the signal component, thereby the amplitude is less.

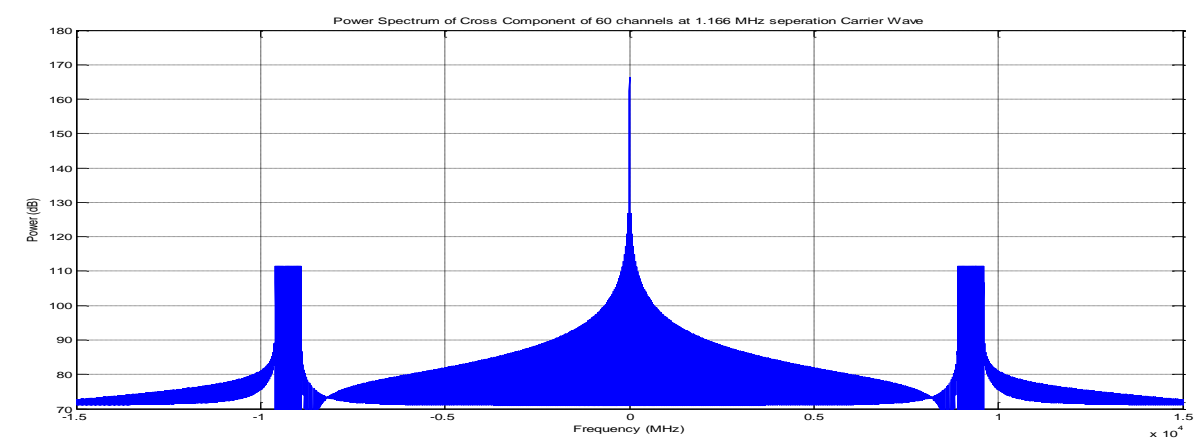

Fig. 14: Plots of power spectrum of cross component of 60 channels at $1.166 \mathrm{MHz}$ carrier separation of an SCM system for NRZ data. 
Observation: The above fig. 14 shows the plot of power spectrum of cross component for 60 channels at 1.166 $\mathrm{MHz}$ carrier separation. Optical bit interference (OBI) is present with noise of the SCM system; thereby the amplitude of the interference plus noise is more.

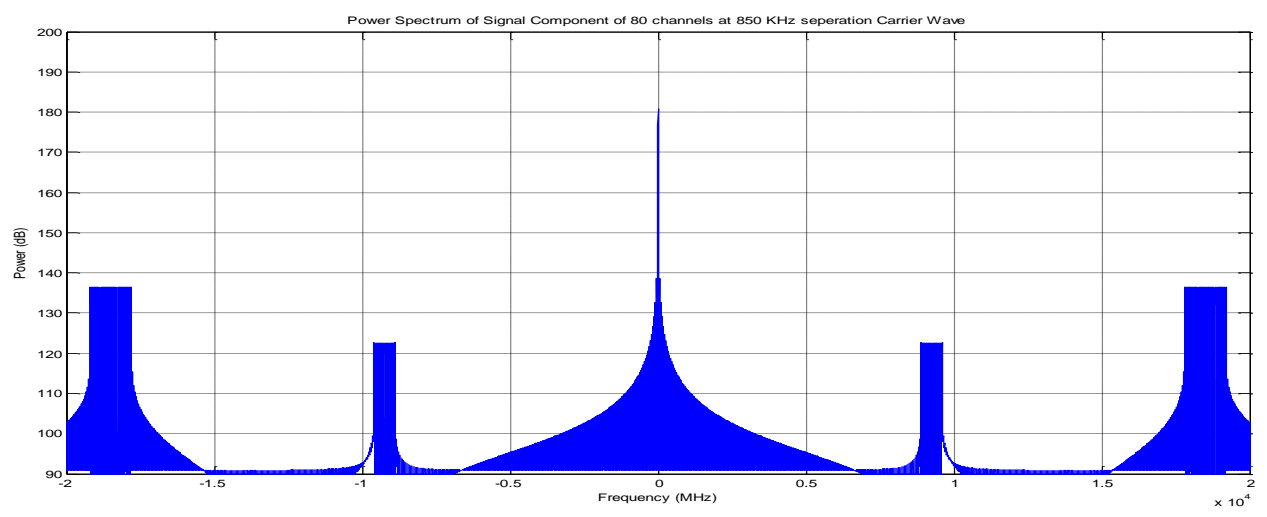

Fig. 15: Plots of power spectrum of signal component of 80 channels at $850 \mathrm{KHz}$ carrier separation of an SCM system for NRZ data.

Observation: The above fig. 15 shows the plot of power spectrum of signal component for 80 channels at 850 $\mathrm{KHz}$ carrier separation. Only noise of the SCM system is present with the signal component, thereby the amplitude is less.

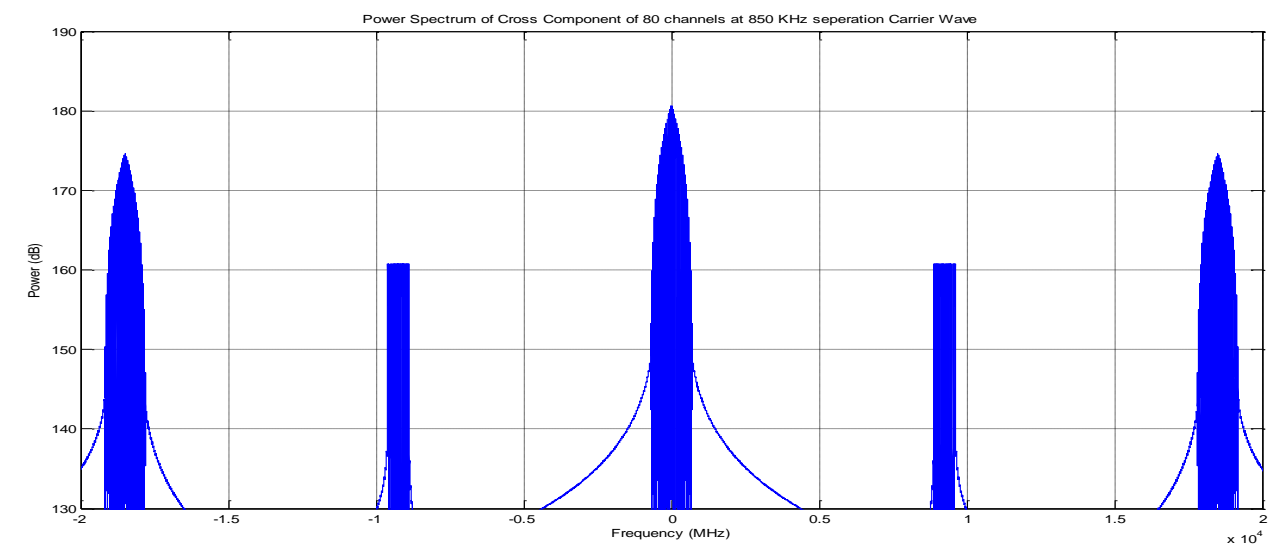

Fig. 16: Plots of power spectrum of cross component of 80 channels at $850 \mathrm{KHz}$ carrier separation of an SCM system for NRZ data.

Observation: The above fig. 16 shows the plot of power spectrum of cross component for 80 channels at 850 $\mathrm{KHz}$ carrier separation. Optical Bit Interference (OBI) is present with noise of the SCM system, thereby the amplitude of the interference plus noise is more and there is significant interference in the plot.

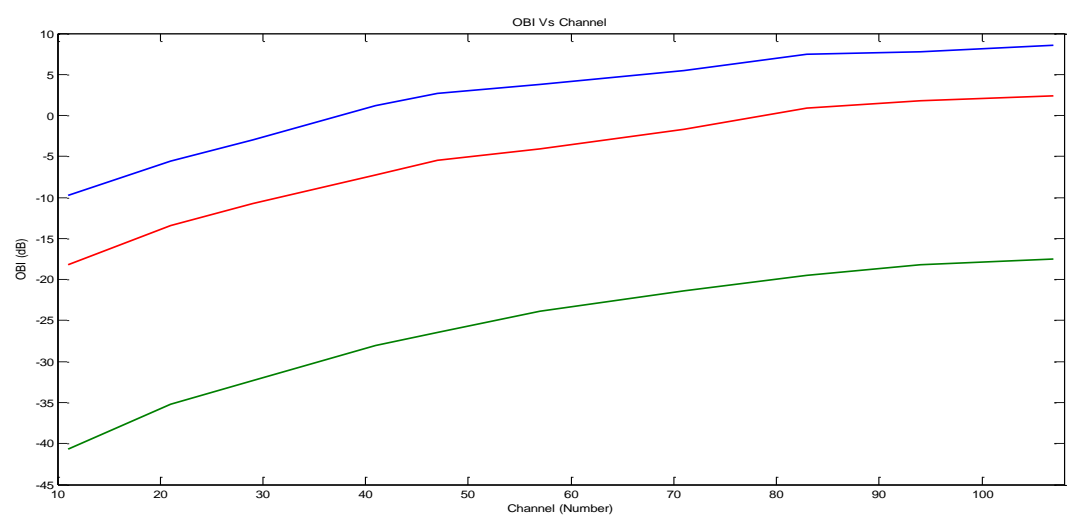

Fig. 17: Plot of Optical Bit Interference (OBI) vs channel number for input power, $P_{i}=1 \mathrm{~dB}, 10 \mathrm{~dB}$ and $20 \mathrm{~dB}$. 
Observation: The fig. 17 shows the plot of OBI vs number of channels for varying input power, which is $1 \mathrm{~dB}$, $10 \mathrm{~dB}$ and $20 \mathrm{~dB}$. It is seen that with the increase of number of channels the optical bit interference also increases. With the increase of input power the OBI also increases for same number of channels.

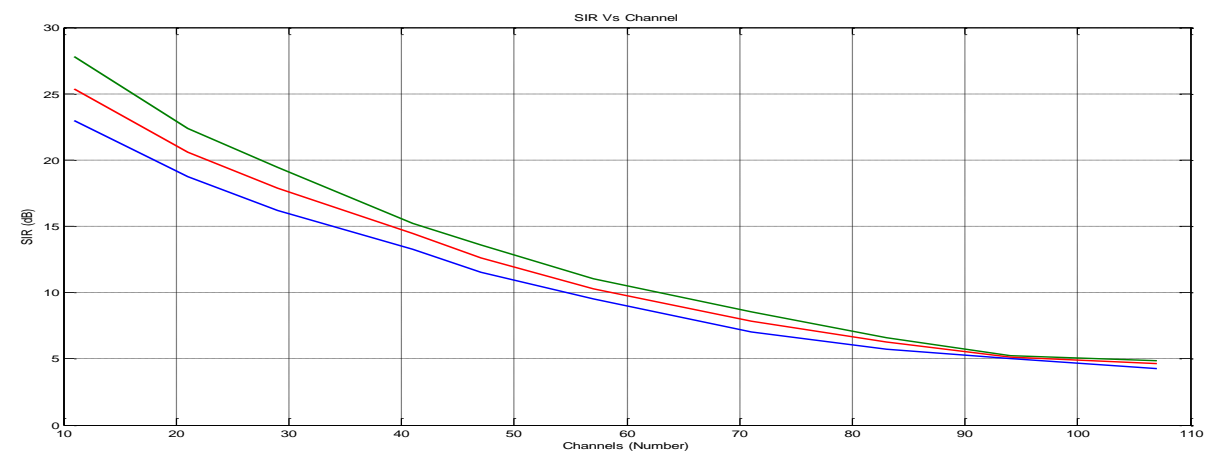

Fig. 18: Plot of Signal to Interference Ratio (SIR) vs channel number for input power, $P_{i}=1 \mathrm{~dB}, 10 \mathrm{~dB}$ and 20 $\mathrm{dB}$.

Observation: The fig. 18 shows the plot of SIR vs number of channels for varying input power, which is $1 \mathrm{~dB}$, $10 \mathrm{~dB}$ and $20 \mathrm{~dB}$. It is seen that with the increase of number of channels the signal to interference ratio also decreases. With the increase of input power the SIR also decreases for same number of channels.

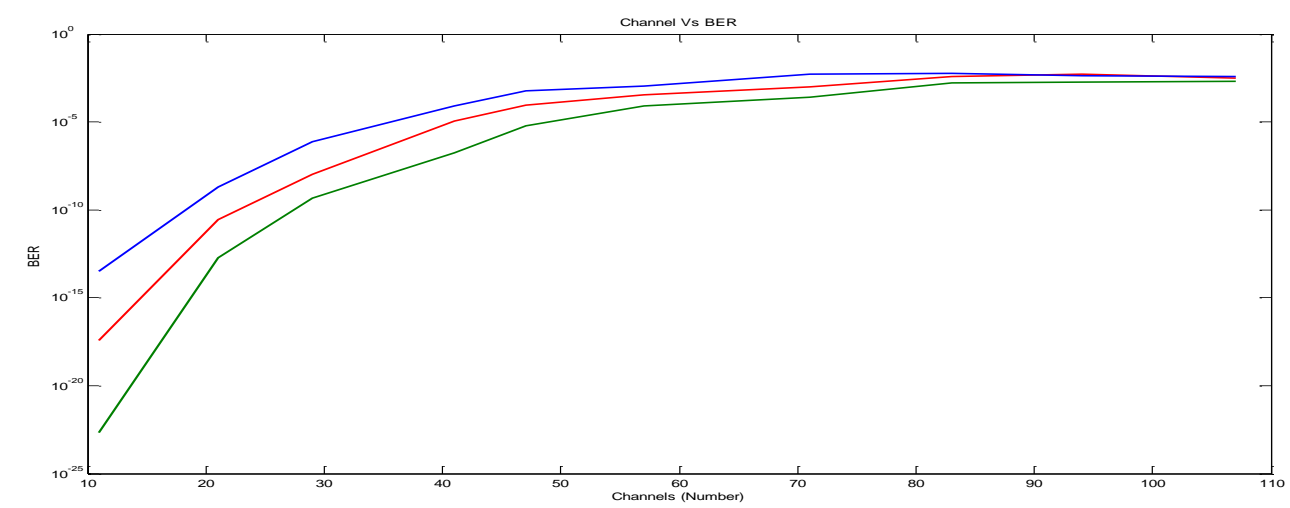

Fig. 19: Plot of Bit Error Rate (BER) vs channel number for input power, $P_{i}=1 \mathrm{~dB}, 10 \mathrm{~dB}$ and $20 \mathrm{~dB}$.

Observation: The fig. 19 shows the plot of BER vs number of channels for varying input power, which is $1 \mathrm{~dB}$, $10 \mathrm{~dB}$ and $20 \mathrm{~dB}$. It is seen that with the increase of number of channels the bit error rate also increases. With the increase of input power the BER also increases for same number of channels.

\subsection{Results}

\section{RESULTS \& DISCUSSIONS}

The bit error rate performance of a SCM system is evaluated considering the effect of crosstalk due to SCM mux/demux. It is found that the system suffers from a power penalty due to crosstalk and the penalty increases with increase in input power for a given number of channels. For a given value of input power, Optical Bit Interference (OBI) increases with the increase of number of channels. For a given value of number of channels OBI produces higher BER. For a given value of input power signal to noise ratio decreases with the increase of number of channels. For a given value of number of channels, with the increase of power, OBI produces higher BER.

\subsection{Discussions}

The number of channels can be increased without significant penalty if the input power is kept low. The number of channels can also be increased if the bandwidth is taken more for more carrier separation. Channel spacing should not be less the $3.5 \mathrm{MHz}$, so that the interference is not significant. Using coherent detection the receiver sensitivity can be improved at minimum input power. Adding a filter in between MUX and DEMUX and maintaining better quality of SCM system components. Using OXC switch based on gates rather than based on space switch. 


\subsection{Overall Design Consideration}

$\checkmark$ Bandwidth: $890 \mathrm{MHz}-960 \mathrm{MHz}$.

$\checkmark$ Channel Spacing: $200 \mathrm{KHz}$.

$\checkmark$ Modulation: QPSK modulation.

$\checkmark$ Line Coding: NRZ input data.

$\checkmark$ Interchannel Spacing and number of Channels: $250 \mathrm{KHz}$.

$\checkmark$ Noise: Noise other than OBI is not considered.

\subsection{Crosstalk Analysis}

A list of different forms of optical crosstalk that may arise in SCM networks was presented in the previous chapter. Optical crosstalk is distinguished based on its spectral difference with the data signal. It was found that optical crosstalk of the same nominal wavelength as the data signal causes a serious degradation in system performance of optical networks because of the presence of crosstalk in SCM system. To calculate the degradation due to crosstalk, an analytical approach was introduced to show relationship among OBI, number of channels, BER, SIR, crosstalk etc. In the presence of a 10 channels, a crosstalk level is less than $-40 \mathrm{~dB}$, whereas for 100 channels it is more than $-20 \mathrm{~dB}$ for the input power $1 \mathrm{~dB}$. As the number of crosstalk sources grows, the requirement on crosstalk performance becomes more stringent.

\subsection{Conclusion}

\section{CONCLUSION \& RECOMMENDATIONS}

The increase of occupied bandwidth due to phase scrambling may exceed the very narrow channel spacing that is required to exploit the available bandwidth efficiently. An even better system performance can be achieved if this technique is applied to optical networks that use low-dispersion fibers. In such networks the effect of spectrum broadening is small and therefore the transmission length can be increased considerably. With respect to the proposed monitoring scheme, it is not recommended to monitor WDM backbone networks with this monitoring scheme since the number of wavelength channels in the backbone networks easily reaches hundreds of channels which is surely beyond the monitoring capability of this adaptive scheme.

\subsection{Recommendations}

In this paper we have restricted our analysis to a linear form of optical crosstalk in a point-to-point SCM optical transmission system. Practical networks consist of several optical nodes that are inter-linked with optical fibers in complex network topologies. Therefore, the crosstalk analysis should be extended to cover crosstalk impairments due to non-linarites in optical fibers and optical amplifiers. Analysis involving all-optical wavelength converters is also desirable, as optical networks become increasingly flexible in terms of wavelength allocations in sub-networks. In addition, we have assumed that, optical pre-detection and electrical post detection filters do not affect the desired signals. This assumption is valid if the filters have an ideal property. Optical amplifiers have been assumed to work in a linear amplification regime. Most of commercial filters and optical amplifiers will introduce a deformation of received signals that can cause the occurrence of inter-symbol interference and amplitude variations. Expanding the method to include inter-symbol interference and gain saturation effects in optical amplifiers will lead to an even better understanding of optical crosstalk.

\section{REFERENCES}

[1]. Arief M., Idrus \& S.M. Alifah, The SCM/WDM system model for radio over fiber communication link, $R F$ and Microwave Conference, IEEE International , pp 344-347, 2-4 December 2008.

[2]. Ira Jacobs, Timothy T. Pratt, John K. Shaw, Rogers H. Stolen \& Anbo Wang, Modeling, analysis and design of subcarrier multiplexing on multimode fiber, Faculty of the Virginia Polytechnic Instituted and State University, March 2003.

[3]. Shijun Xiao \& Andrew M. Weiner, Four-User 3-GHz-Spaced Subcarrier Multiplexing (SCM) Using Optical Direct-Detection via Hyperfine WDM, IEEE Photonics Technology Letters, Vol. 17, No. 10, October 2005.

[4]. Cheng Juang, Shaw Tzuu Huang, Chin Yueh Liu, Wei ChungWang, Tsung Min Hwang, Jonq Juang, and WenWei Lin, Subcarrier Multiplexing by Chaotic Multitone Modulation, IEEE Journal of Quantum Electronics, Vol. 39, No. 10, October 2003.

[5]. M.T. Al-Qdah, H.A. Abdul-Rashid, K. Dimyati, B.M. Ali and M. Khazani, Effect of Optical Beat Interference in

[6]. SCM/WDM Optical Networks in Presence of FWM, KMITL Sci. Tech. J. Vol. 5, No. 3, July-December 2005. 
[7]. Md. Shamim Reza, Md. Maruf Hossain, Adnan Ahmed Chowdhury, S. M. Shamim Reza and Md. Moshiur Rahman, Performance

[8]. Evaluation of SCM-WDM System Using Different Linecoding, Journal of Telecommunications, Vol. 2, Issue 1, April 2010.

[9]. Senior John M., Optical Fiber Communications-Principles and Practice (Prentice-Hall of India, $2^{\text {nd }}$ Edition.)

[10]. Keiser Gerd, Optical Fiber Communications (McGraw-Hill International, $3^{\text {rd }}$ Edition.)

[11]. Simon Haykin, Communication Systems (John Wiley \& Sons Inc., $4^{\text {th }}$ Edition.)

[12]. Simon Haykin, Digital Communications (Wiley India Edition, $1^{\text {st }}$ Edition.)

[13]. Lathi B.P., Modern Digital and Analog Communication Systems (Oxford University Press, $3^{\text {rd }}$ Edition.)

[14]. Yang S. \& Yao J.G., Impact of Crosstalk Induced Beat Noise on the size of Semiconductor Laser Amplifier Based Optical Space Switch Structures, IEEE Photonics Technology Letters, Vol. 4., No. 7 , July 1996.

[15]. Gyselings Tim, Morthier Geert \& Baets Roel, Crosstalk Analysis of Multiwavelength Optical Cross Connects, Journal of Light wave Technology, Vol. 17, No. 8, August 1999.

[16]. Mizuochi Takashi, Kitayama Tadayoshi, Shimizu Katshuhiro \& Ito Atsuyoshi, Interferometric CrosstalkFree Optical Add/Drop Multiplexer Using Mach-Zehnder Based Fiber Gratings, Journal of Light wave Technology, Vol 16, No. 2, February 1998.

[17]. Phase Modulation in Wikipedia, Available: http://en.wikipedia.org/wiki/phase_modulation.

[18]. Timercon website [online]. Available: http://en.timercon.com/BER/

[19]. Accumulated noise in yahoo answer. Available: http://en.answer.com/topic/accumulated_noise/ (2002) The IEEE website. [Online] Available: http://ieee_xplore.com/topic/ effect_of_crosstalk _on_wdm_mux-demux/

[20]. Crosstalk topics. Available: http://www.crosstalkamerica.com/

[21]. Modulation in Wikipedia. Available: http://en.wikipedia.org/wiki/Modulation

[22]. Multiplexing in Wikipedia. Available: http://en.wikipedia.org/wiki/Multiplexing

\section{MATLAB Code}

\section{A. MATLAB Code for 10 Channels}

clc

clear all

close all

$\%$ Sampling frequency

Fs $=65536$;

$\%$ Time vector of 1 second

$\mathrm{t}=0: 1 / \mathrm{Fs}: 1$;

$\mathrm{f}=8900: 70: 9600$;

for $i=1: 65537$

$$
y(i)=20 \text {; }
$$

end

for $\mathrm{i}=1$

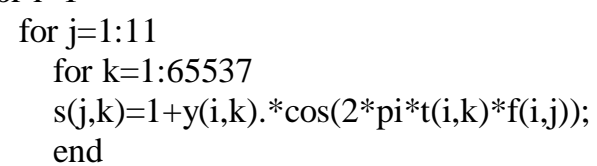

$$
\text { end }
$$

end

for $i=1: 65537$

$\mathrm{x} 10(\mathrm{i})=0$;

$\mathrm{y} 10(\mathrm{i})=0$;

end

for $i=1: 11$

for $\mathrm{j}=1: 65537$

$$
x(i, j)=s(i, j) .^{\wedge} \text {; }
$$$$
\text { end }
$$

end

for $i=1: 65537$

for $\mathrm{j}=1: 11$

\section{APPENDIX}

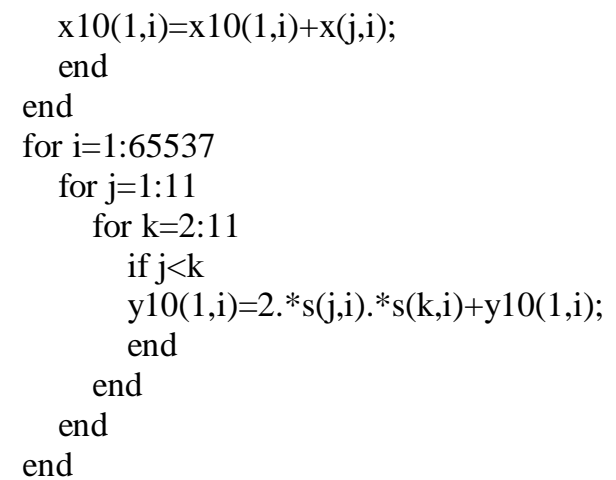

$\%$ Use next highest power of 2 greater than or equal to length $(\mathrm{x})$ to calculate FFT.

$\mathrm{nfft}=2^{\wedge}($ nextpow2 $($ length $(\mathrm{x} 10)))$;

$\%$ Take fft, padding with zeros so that length(fftx)

is equal to $\mathrm{nfft}$

$\mathrm{fftx} 10=\mathrm{fft}(\mathrm{x} 10, \mathrm{nfft})$

ffty10 = fft $(y 10, n f f t)$

$\%$ Calculate the numberof unique points

NumUniquePtsx 10 = $\operatorname{ceil}((\mathrm{nfft}+1) / 2)$;

NumUniquePtsy10 = $\operatorname{ceil}((\mathrm{nfft}+1) / 2)$;

$\%$ FFT is symmetric, throw away second half fftx $10=$ fftx $10(1:$ NumUniquePtsx 10);

ffty10 = ffty10(1:NumUniquePtsy10);

$\%$ Take the magnitude of $\mathrm{fft}$ of $\mathrm{x}$ and scale the $\mathrm{fft}$ so that it is not a function of the length of $\mathrm{x}$ $\mathrm{mx} 10=\mathrm{abs}(\mathrm{fftx} 10) / \mathrm{Fs}$;

my10 = abs(ffty10)/Fs; 
$\%$ Take the square of the magnitude of $\mathrm{fft}$ of $\mathrm{x}$.

$\% \mathrm{mx}=\mathrm{mx} .^{\wedge} 2$

$\%$ Since we dropped half the FFT, we multiply $\mathrm{mx}$ by 2 to keep the same energy.

$\%$ The DC component and Nyquist component, if it exists, are unique and should not be multiplied by 2.

if rem(nfft,2) \% odd nfft excludes Nyquist point $\operatorname{mx} 10(2:$ end $)=\operatorname{mx} 10(2$ :end $) * 2$;

else

$\operatorname{mx} 10(2$ :end -1$)=\operatorname{mx} 10(2$ :end -1$) * 2$;

end

if rem(nfft,2) \% odd nfft excludes Nyquist point $\operatorname{my} 10(2:$ end $)=\operatorname{my} 10(2:$ end $) * 2$;

else

$\operatorname{my} 10(2$ :end -1$)=\operatorname{my} 10(2$ :end -1$) * 2$;

end

$\%$ This is an evenly spaced frequency vector with NumUniquePts points.

fx10 $=((0:$ NumUniquePtsx 10-1)*Fs/nfft $) / 10$; fy $10=((0:$ NumUniquePtsy10-1 $) * \mathrm{Fs} / \mathrm{nfft}) / 10$;

$\%$ Generate the plot, title and labels.

figure(1)

plot(fx10,mx10);

title('Power Spectrum of 10 channels at $7 \mathrm{MHz}$

seperation Carrier Wave');

xlabel('Frequency (MHz)');

ylabel('Power');

axis([ [925 962025$])$

grid on

$\mathrm{F}=\mathrm{f} / 10$;

areamx $10=0$;

areamy $10=0$;

si $=0$;

$\mathrm{ei}=0$;

si $1=0$;

ei $1=0$;

si $=$ find $(\mathrm{f} \times 10==(925-0.1))$

ei $=$ find $(f \times 10==(925+0.1))$

$\mathrm{df}=\mathrm{fx} 10$ (si:ei);

$\mathrm{dmx}=\mathrm{mx} 10$ (si:ei);

areamx $10=\operatorname{trapz}(\mathrm{df}, \mathrm{dmx})$

si $1=$ find $(\mathrm{fx} 10==920.4)$

ei $1=$ find $(\mathrm{f} \times 10==922.6)$

$\mathrm{df}=\mathrm{fx} 10$ (si1:ei1);

$\mathrm{dmy}=\mathrm{mx} 10$ (si1:ei1);

areamy $10=\operatorname{trapz}(\mathrm{df}, \mathrm{dmy})$

snr10=areamx10/areamy10;

snr $10 \mathrm{db}=20 . * \log 10($ snr 10$)$

ber $10=0.5 * \operatorname{erfc}(\operatorname{sqrt}(2 * \operatorname{snr} 10))$

\section{B. MATLAB Code for 100 Channels}

clc

clear all

close all

$\%$ Sampling frequency

Fs = 65536;

$\%$ Time vector of 1 second $\mathrm{t}=0: 1 / \mathrm{Fs}: 1$
title('Power Spectrum of 10 channels at $7 \mathrm{MHz}$ seperation Carrier Wave');

xlabel('Frequency (MHz)');

ylabel('Power');

axis([889 9250 2 0 2])

grid on

figure(2)

plot(fx10,mx10);

title('Power Spectrum of 10 channels at $7 \mathrm{MHz}$ seperation Carrier Wave');

xlabel('Frequency (MHz)');

ylabel('Power');

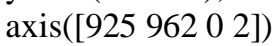

grid on

figure(3)

plot(fy10,my10);

title('Power Spectrum of 10 channels at $7 \mathrm{MHz}$ seperation Carrier Wave');

xlabel('Frequency (MHz)');

ylabel('Power');

axis([889 9250025$])$

grid on

figure(4)

plot(fy10,my10);

$\mathrm{f}=8900: 6.5: 9600$;

for $i=1: 65537$

$y(i)=0.01$;

end

for $i=1$

for $\mathrm{j}=1: 108$

for $\mathrm{k}=1: 65537$

$\mathrm{s}(\mathrm{j}, \mathrm{k})=1+\mathrm{y}(\mathrm{i}, \mathrm{k}) . * \cos \left(2 * \mathrm{pi}^{*} \mathrm{t}(\mathrm{i}, \mathrm{k}) * \mathrm{f}(\mathrm{i}, \mathrm{j})\right)$; end

end

end

for $i=1: 65537$

$\mathrm{x} 100(\mathrm{i})=0$;

$\mathrm{y} 100(\mathrm{i})=0$;

end

for $\mathrm{i}=1: 108$

for $j=1: 65537$

end

end

for $i=1: 65537$

for $\mathrm{j}=1: 108$

$\mathrm{x} 100(1, \mathrm{i})=\mathrm{x} 100(1, \mathrm{i})+\mathrm{x}(\mathrm{j}, \mathrm{i})$;

end

end

for $i=1: 65537$

for $\mathrm{j}=1: 108$

for $\mathrm{k}=2: 108$

if $\mathrm{j}<\mathrm{k}$

$\mathrm{y} 100(1, \mathrm{i})=2 . * \mathrm{~s}(\mathrm{j}, \mathrm{i}) . * \mathrm{~s}(\mathrm{k}, \mathrm{i})+\mathrm{y} 100(1, \mathrm{i})$;

end

end

end

end 
$\%$ Use next highest power of 2 greater than or equal to length(x) to calculate FFT.

$\mathrm{nfft}=2^{\wedge}($ nextpow2(length $\left.(\mathrm{x} 100))\right)$;

$\%$ Take $\mathrm{fft}$, padding with zeros so that length(fftx) is equal to $\mathrm{nfft}$

fftx100 = fft(x100,nfft);

ffty100 = fft $(\mathrm{y} 100, \mathrm{nfft})$;

$\%$ Calculate the numberof unique points

NumUniquePtsx $100=\operatorname{ceil}((\mathrm{nfft}+1) / 2)$;

NumUniquePtsy100 = ceil $((\mathrm{nfft}+1) / 2)$;

$\%$ FFT is symmetric, throw away second half

$\mathrm{fftx} 100=\mathrm{fftx} 100(1:$ NumUniquePtsx 100);

ffty100 = ffty100(1:NumUniquePtsy100);

$\%$ Take the magnitude of $\mathrm{fft}$ of $\mathrm{x}$ and scale the $\mathrm{ftt}$

so that it is not a function of the length of $\mathrm{x}$

$\mathrm{mx} 100=\mathrm{abs}(\mathrm{fftx} 100) / \mathrm{Fs}$;

my100 = abs(ffty100)/Fs;

$\%$ Take the square of the magnitude of fft of $\mathrm{x}$.

$\% \mathrm{mx}=\mathrm{mx} .^{\wedge} 2$

$\%$ Since we dropped half the FFT, we multiply $\mathrm{mx}$

by 2 to keep the same energy.

$\%$ The DC component and Nyquist component, if it exists, are unique and should not be multiplied by

2.

if rem(nfft,2) \% odd nfft excludes Nyquist point

$\operatorname{mx} 100(2:$ end $)=\operatorname{mx} 100(2:$ end $) * 2$;

else

$\mathrm{mx} 100(2$ :end -1$)=\operatorname{mx} 100(2$ :end -1$) * 2$;

end

if rem(nfft,2) \% odd nfft excludes Nyquist point my100 $(2$ :end $)=\operatorname{my} 100(2$ :end $) * 2$;

else

$\operatorname{my} 100(2$ :end -1$)=\operatorname{my} 100(2$ :end -1$) * 2$;

end

$\%$ This is an evenly spaced frequency vector with NumUniquePts points.

fx $100=((0:$ NumUniquePtsx $100-1) * \mathrm{Fs} / \mathrm{nfft}) / 10$;

fy100 $=((0:$ NumUniquePtsy100-1 $) * \mathrm{Fs} / \mathrm{nfft}) / 10$;

$\%$ Generate the plot, title and labels.

figure(1)

plot(fx100,mx100);

title('Power Spectrum of 100 channels at $700 \mathrm{Khz}$

seperation Carrier Wave');

xlabel('Frequency (MHz)');

ylabel('Power');

axis([889 92502 2 0 )

grid on

figure(2)

plot(fx100,mx100);

title('Power Spectrum of 100 channels at $700 \mathrm{Khz}$

seperation Carrier Wave');

xlabel('Frequency $(\mathrm{MHz})$ ');

ylabel('Power');

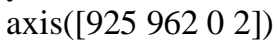

grid on

figure(3)

plot(fy100,my100);

title('Power Spectrum of 100 channels at $700 \mathrm{Khz}$

seperation Carrier Wave');
xlabel('Frequency (MHz)');

ylabel('Power');

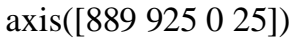

grid on

figure(4)

plot(fy100,my100);

title('Power Spectrum of 100 channels at $700 \mathrm{Khz}$

seperation Carrier Wave');

xlabel('Frequency (MHz)');

ylabel('Power');

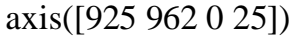

grid on

$\mathrm{F}=\mathrm{f} / 10$;

areamx100 $=0$;

areamy $100=0$;

si $=0$;

ei $=0$;

si $1=0$;

ei $1=0$;

$\%$ for $\mathrm{i}=1: 108$

$\%$ si $=$ find $(\mathrm{f} \times 100==(\mathrm{F}(\mathrm{i})-0.1))$

$\%$ ei $=$ find $(\mathrm{f} \times 100==(\mathrm{F}(\mathrm{i})+0.1))$

$\% \mathrm{df}=\mathrm{fx} 100$ (si:ei);

$\% \mathrm{dmx}=\mathrm{mx} 100$ (si:ei);

$\%$ areamx $100=\operatorname{areamx} 100+\operatorname{trapz}(\mathrm{df}, \mathrm{dmx})$;

$\%$ end

si $=$ find $($ f $\times 100==(890-0.1))$

ei $=$ find $(\mathrm{f} \times 100==(890+0.1))$

$\mathrm{df}=\mathrm{fx} 100$ (si:ei);

$\mathrm{dmx}=\mathrm{mx} 100$ (si:ei);

areamx $100=\operatorname{trapz}(\mathrm{df}, \mathrm{dmx})$

si $1=$ find $(f \times 100==890.1)$

ei $1=$ find $(f \times 100==890.55)$

$\mathrm{df}=\mathrm{fx} 100$ (si1:ei1);

dmy=mx100(si1:ei1);

areamy $100=\operatorname{trapz}(\mathrm{df}, \mathrm{dmy})$

snr $100=$ areamx 100/areamy100;

$\operatorname{snr} 100 \mathrm{db}=20 . * \log 10(\operatorname{snr} 100)$

ber $100=0.5 * \operatorname{erfc}(\operatorname{sqrt}(2 * \operatorname{snr} 100))$

C. MATLAB Code for Channel vs SNR \& BER

clc

clear all

close all

$\mathrm{p}=\left[\begin{array}{llll}1 & 5 & 10 & 20\end{array}\right]$;

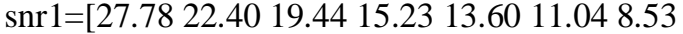

$6.615 .254 .86]$;

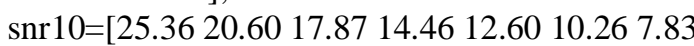

$6.295 .104 .64]$;

snr20=[22.9497 18.758916 .202913 .250511 .5074

9.5336 7.052 5.7121 5.0435 4.2542];

channel=[11 2129414757718394 107];

BER1=[2.10E-23 1.93E-13 4.53E-10 1.79E-07

6.09E-06 7.95E-05 2.68E-04 0.0017 0.0019

$0.0021]$

BER5=[5.55E-21 1.75E-12 1.70E-09 1.67E-06

9.57E-06 5.65E-05 3.30E-04 0.0018 0.0024 0.004]; 
BER10=[3.64E-18 2.90E-11 1.10E-08 1.14E-05 8.81E-05 3.54E-04 9.42E-04 0.004 0.0054 0.003]; $\mathrm{BER} 20=[3.31 \mathrm{E}-14$ 1.95E-09 7.79E-07 7.96E-05 5.75E-04 1.12E-03 5.38E-03 0.0057 0.0043 $0.0039]$;

OBI1 $=[-40.63-35.24-32.29-28.07-26.47-23.89-$ $21.41-19.44-18.24-17.54]$;

OBI5 $=[-25.63-20.53-17.69-14.13-12.06-9.93-$ $8.06-5.39-4.26-3.56]$;

OBI10 $=[-18.21-13.44-10.70-7.28-5.42-4.06-$ $1.640 .881 .772 .43]$

OBI20=[-9.77 $-5.55-2.981 .23 \quad 2.73 .755 .447 .50$

7.81 8.52];

ber11=[2.10E-23 5.55E-21 3.64E-18 3.31E-14]; ber21=[1.93E-13 1.75E-12 2.90E-11 1.95E-09];

hold on

plot(channel,OBI20);

title('OBI Vs Channel');

xlabel('Channel (Number)');

ylabel('OBI $\left.(\mathrm{dB})^{\prime}\right)$;

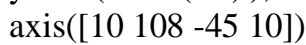

grid on

figure(2)

plot(channel,snr1);

hold on

plot(channel,snr10);

hold on

plot(channel,snr20);

title('SIR Vs Channel');

xlabel('Channels (Number)');

ylabel('SIR (dB)');

$\%$ axis([889 925002$])$

grid on

figure(3)

semilogy(channel,BER1);

hold on

semilogy(channel,BER5);

hold on

semilogy(channel,BER10);

hold on

semilogy(channel,BER20);

title('Channel Vs BER');

xlabel('Channels (Number)');
ber29=[4.53E-10 1.70E-09 1.10E-08 7.79E-07] ber41 $=[1.79 \mathrm{E}-07$ 1.67E-06 1.14E-05 7.96E-05]; ber47 $=[6.09 \mathrm{E}-06$ 9.57E-06 8.81E-05 5.75E-04]; ber57 $=[7.95 \mathrm{E}-05$ 5.65E-05 3.54E-04 1.12E-03]; ber71 $=[2.68 \mathrm{E}-04$ 3.30E-04 9.42E-04 5.38E-03]; ber $83=\left[\begin{array}{lllll}0.0017 & 0.00180 & 0.004 & 0.0057\end{array}\right]$;

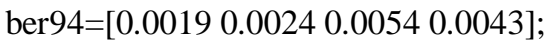

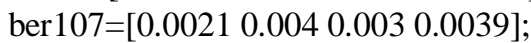
figure(1)

plot(channel,OBI1); hold on plot(channel,OBI5); hold on plot(channel,OBI10);

ylabel('BER ');

$\%$ axis([889 925002$])$

grid on

figure(4)

semilogy(p,ber11);

hold on

semilogy(p,ber21);

hold on

semilogy(p,ber29);

hold on

semilogy(p,ber41);

hold on

semilogy(p,ber47);

hold on

semilogy(p,ber57);

hold on

semilogy(p,ber71);

hold on

semilogy(p,ber83);

hold on

semilogy(p,ber94);

hold on

semilogy(p,ber107);

title('Channel Vs BER');

xlabel('Channels (Number)');

ylabel('BER ');

$\% \operatorname{axis}\left(\left[\begin{array}{lllll}889 & 925 & 0 & 2\end{array}\right]\right)$

grid on 